\title{
Experiencia en la Formación y Operación del Cuerpo Académico ITESI CA-017 Ingeniería de Software
}

\section{Experience in Training and Operation of the Research Group ITESI CA-017 Software Engineering}

TORRES-FRAUSTO, David Antonio $\dagger^{*}$, VILLEGAS-TÉLLEZ, Rodrigo y LOZANO-VÁZQUEZ, Ángel Gerardo

Instituto Tecnológico Superior de Irapuato. Carretera Irapuato-Silao Km. 12.5 Col. El Copal C.P. 36821 Irapuato, Guanajuato, México. Coordinación de Ingeniería en Informática e Ingeniería en Sistemas Computacionales

ID $1^{\text {er }}$ Autor: David Antonio, Torres-Frausto / ORC ID: 0000-0002-1031-2302, Researcher ID Thomson: S-6623-2018, CVU CONACYT ID: 602438

ID $1^{\mathrm{er}}$ Coautor: Rodrigo, Villegas-Téllez, / ORC ID: 0000-0002-8450-5027, Researcher ID Thomson: S-4822-2018, CVU CONACYT ID: 602492

ID $2^{\text {do }}$ Coautor: Ángel Gerardo, Lozano-Vázquez / ORC ID: 0000-0003-0560-6442, Researcher ID Thomson: S-4819-2018, CVU CONACYT ID: 334033

DOI: $10.35429 / J C S .2019 .8 .3 .14 .17$

Recibido: 12 de Marzo, 2019; Aceptado 30 de Junio, 2019

\begin{abstract}
Resumen
El presente artículo tiene como finalidad exponer las experiencias obtenidas en la formación del Cuerpo Académico ITESI CA-017 Ingeniería de Software, el cual integra a profesores de las carreras de Ingeniería Informática e Ingeniería en Sistemas Computacionales del Instituto Tecnológico Superior de Irapuato. En gran parte, el camino recorrido por los miembros del cuerpo académico se ha basado en el sentido de responsabilidad y compromiso que cada uno ha adoptado; lo que ha permitido que se genere un ambiente de confianza idóneo para la colaboración y el trabajo en equipo. Como parte de la experiencia que se comparte en el presente trabajo, se menciona desde la integración, el trámite para la obtención de reconocimiento como Cuerpo Académico en Formación por parte de PRODEP, la incorporación de nuevos miembros; hasta la distribución del trabajo y la colaboración en proyectos de investigación y generación de publicaciones para participación en foros, tanto nacionales como internacionales. $\mathrm{La}$ experiencia presentada, busca contribuir a que otros profesores se integren como grupos de investigación y colaboren entre ellos; e incluso con el Cuerpo Académico ITESI CA-017 Ingeniería de Software.
\end{abstract}

Cuerpo Académico, Investigación, Colaboración

\begin{abstract}
The purpose of this article is to present the experiences obtained in the formation of the Research Group ITESI CA-017 of Software Engineering, which is formed with professors of the Computer Engineering and Computer Systems Engineering from the Instituto Tecnológico Superior de Irapuato. Largely, the path taken by the members of the academic body has been based on the sense of responsibility and commitment that each one has adopted; this has allowed the creation of an environment of trust suitable for collaboration and teamwork. As part of the experience that is shared in this work, is mentioned from the integration, the procedure for obtaining recognition as a Research Group in Training by PRODEP, the incorporation of new members; finishing in the distribution of work and collaboration in research projects and generation of publications for participation in national and international forums. The propuse of the presented experience is to help other professors integrate as research groups and collaborate with each other; and even with the Research Group ITESI CA-017 Software Engineering.
\end{abstract}

Research Group, Research, Collaboration

Citación: TORRES-FRAUSTO, David Antonio, VILLEGAS-TÉLLEZ, Rodrigo y LOZANO-VÁZQUEZ, Ángel Gerardo. Experiencia en la Formación y Operación del Cuerpo Académico ITESI CA-017 Ingeniería de Software. Revista de Simulación Computacional. 2019. 3-8: 14-17

\footnotetext{
* Correspondencia al Autor: (datorres@itesi.edu.mx)

$\dagger$ Investigador contribuyendo como primer autor.
} 


\section{Introducción}

Para lograr una educación de calidad, resulta indispensable un proceso continuo de evaluación y retroalimentación. Atrás quedaron las ideas de que un profesor sólo se limita a impartir clase. Su desempeño actual requiere el cumplimiento de otras funciones sustantivas, además de la docencia, mediante las cuales pueda otorgar valor agregado a la institución de educación superior (IES) donde labora, y sobre todo a los alumnos que cursan alguno de los planes de estudios que ésta institución oferta. Estas funciones se pueden categorizar en cuatro grandes rubros: docencia, investigación, gestión académica y vinculación.

El Programa para el Desarrollo Profesional Docente, para el Tipo Superior (PRODEP) surge buscando profesionalizar a los Profesores de Tiempo Completo (PTC) para que alcancen las capacidades de investigacióndocencia, desarrollo tecnológico e innovación y con responsabilidad social, se articulen y consoliden en cuerpos académicos y con ello generen una nueva comunidad académica capaz de transformar su entorno (Secretaría de Educación Pública, 2019).

De acuerdo con PRODEP, un Cuerpo Académico (CA) es un conjunto de profesoresinvestigadores que comparten una o más líneas de estudio, cuyos objetivos y metas están destinados a la generación y/o aplicación de nuevos conocimientos. Además, por el alto grado de especialización que alcanzan en conjunto al ejercer la docencia, logran una educación de buena calidad. Los cuerpos académicos sustentan las funciones académicas institucionales y contribuyen a integrar el sistema de educación superior del país.

Los CA constituyen un sustento indispensable para la formación de profesionales y expertos. Dada la investigación que realizan, son un instrumento de profesionalización del profesorado y de su permanente actualización, por lo tanto, favorecen una plataforma sólida para enfrentar el futuro cada vez más exigente en la formación de capital humano, situación que les permite erigirse como las células de la academia y representar a las masas críticas en las diferentes áreas del conocimiento que regulan la vida académica de las Instituciones de Educación Superior (Secretaría de Educación Pública, 2019).
Si bien no es requisito indispensable que cada profesor de tiempo completo forme parte de un Cuerpo Académico, realizar trabajo colectivo resulta de gran utilidad y enriquecimiento académico.

\section{Metodología}

El cuerpo académico ITESI CA-017 Ingeniería de Software nace con la finalidad de reunir las contribuciones de varios profesores de tiempo completo de las carreras de Ingeniería Informática e Ingeniería en Sistemas Computacionales del Instituto Tecnológico Superior de Irapuato. Su evolución y crecimiento hasta el momento, puede explicarse en las siguientes etapas:

\section{Formación}

El punto de inicio en la formación del cuerpo académico se da a raíz de que dos de los miembros obtienen el reconocimiento a perfil deseable y apoyo que otorga la Dirección de Superación Académica, en la convocatoria 2016. Es aquí donde se cae en cuenta que varias de las actividades que la convocatoria solicita que se evidencien, ya son realizadas por los profesores de forma cotidiana; lo que los impulsa a buscar un siguiente paso, la formación de un cuerpo académico.

La búsqueda de un tercer miembro, requisito indispensable para la formación de un cuerpo académico, lleva a analizar diferentes perfiles de compañeros. Cabe señalar que no bastó con analizar perfiles profesionales, si no que tuvo un mayor peso la afinidad de personalidades y la disposición para el trabajo y el compromiso.

De esta forma, se comienzan a realizar reuniones de trabajo para informar sobre las actividades realizadas y planificar las actividades futuras, a fin de revisar la manera en que cada uno de los miembros pudiera contribuir, de alguna manera, en el desarrollo personal y profesional del resto de los miembros.

\section{Formalización y reconocimiento}

Al publicarse la convocatoria 2017 para el reconocimiento de cuerpos académicos de PRODEP, se decide formalizar la conformación del cuerpo académico. 
Cabe señalar que, aunque en ese momento la productividad del tercer miembro del cuerpo académico no era basta, se evidenció que se había iniciado en ese año a generar varios productos que, en un futuro, resultarían valiosos para aportar al cuerpo académico (Tabla 1).

\begin{tabular}{|c|c|}
\hline Miembro del CA & $\begin{array}{l}\text { Producción } \\
\text { Académica }\end{array}$ \\
\hline $\begin{array}{l}\text { Elizabeth Gabriela Vargas } \\
\text { Espinoza }\end{array}$ & 4 \\
\hline Rodrigo Villegas Téllez & 14 \\
\hline David Antonio Torres Frausto & 13 \\
\hline
\end{tabular}

Tabla 1 Producción Académica 2015-2017 por miembro del CA

De esta forma, se han realizado diversos trabajos en colaboración, así como proyectos de investigación y desarrollo tecnológico en diferentes áreas de informática y computación.

\section{Crecimiento}

Una vez obtenido el reconocimiento por parte de PRODEP como cuerpo académico en formación, se ha continuado trabajando en diversos proyectos. Incluso, se han integrado dos nuevos miembros pertenecientes a la carrera de Ingeniería en Sistemas Computacionales, logrando nutrir al cuerpo académico desde diversas áreas afines a la Ingeniería de Software, siendo actualmente formado por cinco profesores de tiempo completo (Tabla 2).

\begin{tabular}{|c|c|}
\hline Nombre & Carrera de adscripción \\
\hline $\begin{array}{l}\text { María Guadalupe } \\
\text { Amézquita Delgado }\end{array}$ & $\begin{array}{ll}\text { Ingeniería en } & \text { Sistemas } \\
\text { Computacionales } & \\
\end{array}$ \\
\hline $\begin{array}{l}\text { Ángel Gerardo Lozano } \\
\text { Vázquez }\end{array}$ & $\begin{array}{ll}\text { Ingeniería en } & \text { Sistemas } \\
\text { Computacionales } & \\
\end{array}$ \\
\hline $\begin{array}{l}\text { David Antonio Torres } \\
\text { Frausto }\end{array}$ & Ingeniería Informática \\
\hline $\begin{array}{l}\text { Elizabeth Gabriela } \\
\text { Vargas Espinoza }\end{array}$ & $\begin{array}{ll}\text { Ingeniería en } & \text { Sistemas } \\
\text { Computacionales } & \\
\end{array}$ \\
\hline Rodrigo Villegas Téllez & Ingeniería Informática \\
\hline
\end{tabular}

Tabla 2 Miembros actuales del CA

Más recientemente, en la convocatoria 2019 para obtención de reconocimiento a perfil deseable y apoyo, los tres profesores que ingresaron a la convocatoria, obtuvieron su reconocimiento; y los otros dos obtuvieron un veredicto favorable a la renovación del reconocimiento. De ésta forma, se tiene que el $100 \%$ de los profesores que integra actualmente el cuerpo académico tienen el reconocimiento a perfil deseable PRODEP.

\begin{tabular}{|l|c|}
\hline \multicolumn{1}{|c|}{ Sección } & Número de registros \\
\hline Dirección individualizada & 20 \\
\hline Producción académica & 27 \\
\hline Proyectos de investigación & 1 \\
\hline
\end{tabular}

Tabla 3 Producción del CA

Trabajo colaborativo

Aunque se ha logrado la integración de los miembros del CA, es necesario continuar con el trabajo realizado hasta el momento, en la búsqueda de mejorar el desempeño de manera individual y como grupo de trabajo.

\section{Conclusiones}

En términos generales, la evaluación que puede realizarse al interior del CA demuestra el trabajo colaborativo e individual de cada uno de sus miembros. Sin embargo, resultan también varias áreas de oportunidad que aún faltan por trabajar, las cuales se mencionan a continuación:

\section{Trabajo colaborativo con otros CA's}

Si bien ya se han establecido vínculos con cuerpos académicos de otras instituciones pertenecientes al Tecnológico Nacional de México, tales como el Instituto Tecnológico Superior de Zacatecas Occidente y el Instituto Tecnológico Superior de Zacatecas Sur, resulta vital consolidar los proyectos en los que se tendría dicha colaboración.

\section{Grado de consolidación}

Actualmente el cuerpo académico permanece en formación; y de acuerdo con los requisitos que marca PRODEP para aspirar a un grado de consolidación mayor, es necesario contar con la colaboración de un miembro que tenga estudios de doctorado. De esta forma, se plantea la necesidad a la institución de la contratación de un profesor de tiempo completo adscrito a alguna de las carreras afines al cuerpo académico, y que contribuya con su experiencia a enriquecer los trabajos realizados. 
Participación en convocatorias externas de apoyo a proyectos de investigación y desarrollo tecnológico

Aunque se ha participado en la convocatoria para el fortalecimiento de cuerpos Académicos del propio PRODEP, no se ha tenido suerte; ya que a pesar de haber sido de los proyectos mejor evaluados en la convocatoria 2018, no se tuvieron los recursos necesarios para contar con dicho apoyo.

En el presente año, se decidió participar con el mismo proyecto buscando el apoyo en la convocatoria de apoyo a proyectos de investigación propia de la institución de adscripción, y se está en la espera de los resultados. Sin embargo, resulta necesario buscar otras fuentes de financiamiento que impulsen el desarrollo de este tipo de proyectos.

\section{Registro de autoría (INDAUTOR)}

Una parte importante que ha logrado impulsar el cuerpo académico, ha sido el número de registros de autoría en la institución por el software generado en los últimos años, logrando tener un total de tres registros y otros dos en trámite, en un periodo de dos años.

De esta manera, se asegura el impacto que tienen los proyectos de desarrollo tecnológico en la productividad académica, logrando no solo resultar en la publicación de los resultados, sino también en el correspondiente registro ante INDAUTOR.

\section{Referencias}

Miembros del Cuerpo Académico ITESI CA017 Ingeniería de Software. (2019). Información de Currículum PRODEP. Obtenido de https://promepca.sep.gob.mx/solicitudca/

Secretaría de Educación Pública. (2019). Cuerpos académicos reconocidos por PRODEP. Obtenido de http://promep.sep.gob.mx/CA1/

Secretaría de Educación Pública. (2019). Programa para el Desarrollo Profesional Docente, para el Tipo Superior (PRODEP). Obtenido de https://www.dgesu.ses.sep.gob.mx/PRODEP.ht $\mathrm{m}$ 